\section{TATRA \\ MOUNTaiNS \\ Mathematical Publications}

DOI: $10.2478 / \mathrm{tmmp}-2020-0002$

Tatra Mt. Math. Publ. 75 (2020), 23-32

\title{
BAYESIAN ESTIMATE OF PARAMETERS FOR ARMA MODEL FORECASTING
}

\author{
ZuL AMRY \\ Department of Mathematics, Faculty of Mathematics and Natural Sciences, State University \\ of Medan, Medan, INDONESIA
}

\begin{abstract}
This paper presents a Bayesian approach to finding the Bayes estimator of parameters for ARMA model forecasting under normal-gamma prior assumption with a quadratic loss function in mathematical expression. Obtaining the conditional posterior predictive density is based on the normal-gamma prior and the conditional predictive density, whereas its marginal conditional posterior predictive density is obtained using the conditional posterior predictive density. Furthermore, the Bayes estimator of parameters is derived from the marginal conditional posterior predictive density.
\end{abstract}

\section{Introduction}

Bayesian inference is a method of analysis that combines information collected from experimental data with the knowledge that one has prior to performing the experiment. The Bayesian approach in general requires an explicit formulation of a model and conditioning on known quantities, in order to draw inferences about the unknown. The forecasting model is the most often recognized as Bayesian forecasting when a probability distribution is used to describe uncertainty regarding the unknown parameters and when Bayes theorem is applied. For forecasting problems, Bayesian approach combining all the information and sources of uncertainty into a predictive distribution of the future values to estimate the parameters in model. The main idea of Bayesian forecasting is the predictive distribution of the future given past data following directly from the joint probabilistic model. The predictive distribution is derived from the sampling predictive density, weighted by the posterior distribution.

(C) 2020 Mathematical Institute, Slovak Academy of Sciences.

2010 Mathematics Subject Classification: 62C10.

Keywords: ARMA model, Bayes estimator, normal-gamma prior, quadratic loss function. Licensed under the Creative Commons Attribution-NC-ND4.0 International Public License. 
This paper is done refering to Li u discussion on Bayesian analysis for one-step ahead forecast in ARMA model. The other papers related to this research are Fan and $\mathrm{Yao}, \mathrm{Kleibergen}$ and $\mathrm{Hoek}$, and Ut u r bey also discussed the Bayesian analysis for ARMA model. This paper focuses on finding the mathematical expression of the Bayes estimator of parameters for the ARMA model forecasting under normal-gamma prior assumption with a quadratic loss function.

\section{Materials and methods}

The materials in this paper are some theories in mathematics and statistics such as the ARMA model, Bayes theorem, repeated integration, gamma distribution and the multivariate t-distribution. The method is a study of literature by applying the Bayesian analysis under normal-gamma prior assumption.

Definition 2.1. The ARMA $(p, q)$ model is defined by

$$
y_{t}=\sum_{i=1}^{p} \phi_{i} y_{t-i}+\sum_{j=1}^{q} \theta_{j} e_{t-j}+e_{t}
$$

where $\left\{e_{t}\right\}$ is a sequence of iid normal random variables with

$$
e_{t} \sim N\left(0, \tau^{-1}\right), \tau>0
$$

and unknowns $\phi_{i}$ and $\theta_{j}$ are parameters.

Definition 2.2. The Bayes theorem can be expressed as

$$
(\text { posterior }) \propto(\text { prior }) \times(\text { likelihood }) .
$$

Definition 2.3. A positive random quantity $\phi$ is said to have a gamma distribution with parameter $n>0$ and $d>0$ if it has the probability density function

$$
p(\phi)=\frac{d^{n}}{\Gamma(n)} d^{n-1} \exp (-\phi d)
$$

the mean is $E(\phi)=\frac{n}{d}$ and the variance is $\operatorname{Var}(\phi)=\frac{n}{d^{2}}$.

Definition 2.4. A random $p$-vector $\underline{X}$ is said to have a joint student-t distribution on $n$ degrees of freedom with mode $\mu$ and scale matrix $\Omega$ if it has the probability density function

$$
p(\underline{X})=\frac{\Gamma\left(\frac{n+p}{2}\right) n^{\frac{n+p-1}{2}}}{\Gamma\left(\frac{n}{2}\right) \pi^{\frac{p}{2}}|\Omega|^{\frac{p}{2}}}\left(n+(x-\mu)^{T} \Omega^{-1}(x-\mu)\right)^{-\frac{n+p}{2}} .
$$




\section{Construction of the estimator}

The $k$-step-ahead point forecast of $y_{n+k}$ is defined by

$$
\hat{y}(k)=E\left(y_{n+k} \mid S_{n}^{*}\right),
$$

where

$$
S_{n}^{*}=\left(y_{1}, y_{2}, \ldots, y_{n+k-1}\right) .
$$

Using the equation (1) one obtains residuals as

$$
e_{t}=y_{t}-\sum_{i=1}^{p} \phi_{i} y_{t-i}-\sum_{j=1}^{q} \theta_{j} e_{t-j}
$$

and by conditioning the first $p$ observations and letting $e_{p}=e_{p-1}=\cdots=e_{r}=0$, where $r=\min (0, p+1-q)$, one may approximate by $\mathrm{B}$ ox and $\mathrm{J}$ e $\mathrm{nk}$ in s in [5], the likelihood function for parameters $\Psi=\left(\phi_{1}, \phi_{2}, \ldots, \phi_{p}, \theta_{1}, \theta_{2}, \ldots, \theta_{q}\right)$ and $\tau$ based on $S_{n}^{*}$ is

$$
L\left(\Psi, \tau \mid S_{n}^{*}\right) \propto \tau^{\frac{(n+k-1)-p}{2}} \exp \left(-\frac{\tau}{2}\left(\sum_{t=p+1}^{n+k-1}\left(y_{t}-\sum_{i=1}^{p} \phi_{i} y_{t-i}-\sum_{j=1}^{q} \theta_{j} e_{t-j}\right)^{2}\right)\right) .
$$

The equation (7) can be expressed as

$$
\begin{array}{r}
L\left(\Psi, \tau \mid S_{n}^{*}\right) \propto \tau^{\frac{(n+k-1)-p}{2}} \exp \left(-\frac{\tau}{2}\left(\sum_{t=p+1}^{n+k-1} y_{t}^{2}-2 \Psi^{T} \sum_{t=p+1}^{n+k-1} y_{t} B_{t-1}+\right.\right. \\
\left.\left.\sum_{t=p+1}^{n+k-1}\left(\Psi^{T} B_{t-1}\right)^{2}\right)\right),
\end{array}
$$

where $B_{t}=\left(y_{t}, y_{t-1}, \ldots, y_{t+1-p}, e_{t}, e_{t-1}, \ldots, e_{t+1-q}\right)$. By letting

$$
U=\left(\begin{array}{cccc}
y_{p} & y_{p+1} & \cdots & y_{n+k-2} \\
y_{p-1} & y_{p} & \cdots & y_{n+k-3} \\
\vdots & \vdots & \vdots & \vdots \\
y_{1} & y_{2} & \cdots & y_{n+k-1-p} \\
e_{p} & e_{p+1} & \cdots & e_{n+k-2} \\
e_{p-1} & e_{p} & \cdots & e_{n+k-3} \\
\vdots & \vdots & \vdots & \vdots \\
e_{1} & e_{2} & \cdots & e_{n+k-1-p}
\end{array}\right), \quad X_{0}=\left(\begin{array}{c}
y_{p+1} \\
y_{p+2} \\
\vdots \\
y_{n+k-1}
\end{array}\right) \text {, }
$$

where $e_{t}=y_{t}-\sum_{i=1}^{p} \tilde{\phi}_{i} y_{t-i}-\sum_{j=1}^{q} \tilde{\theta}_{j} \hat{e}_{t-j}, t=p+1, p+2, \ldots, n, \tilde{\phi}_{i}$ and $\tilde{\theta}_{j}$ are maximum likelihood estimator of $\tilde{\phi}_{i}$ and $\tilde{\theta}_{j} \cdot e_{t}, e_{t-1}, \ldots, e_{t-q}$ is obtained via

$$
e_{t}=y_{t}-\tilde{\Psi}^{T} B_{t-1},
$$

where $\tilde{\Psi}=\left(\tilde{\phi}_{1}, \tilde{\phi}_{2}, \ldots, \tilde{\phi}_{p}, \tilde{\theta}_{1}, \tilde{\theta}_{2}, \ldots, \tilde{\theta}_{q}\right)$. 
From the likelihood function in equation (8) one can obtain:

$$
\begin{aligned}
\sum_{t=p+1}^{n+k-1} y_{t} B_{t-1}= & y_{p+1} B_{p}+y_{p+2} B_{p+1}+\cdots+y_{n+k-1} B_{n+k-2} \\
= & y_{p+1}\left(y_{p}, y_{p-1}, \ldots, y_{1}, e_{p}, e_{p-1}, \ldots, e_{p+1-q}\right) \\
& +y_{p+2}\left(y_{p+1}, y_{p}, \ldots, y_{2}, e_{p+1}, e_{p}, \ldots, e_{p+2-q}\right)+\ldots \\
& +y_{n+k-1}\left(y_{n+k-2}, y_{n+k-3}, \ldots, y_{n-k-1-p},\right. \\
& \left.e_{n+k-2}, e_{n+k-3}, \ldots, e_{n+k-1-q}\right) \\
\sum_{t=p+1}^{n+k-1}\left(\Psi^{T} B_{t-1}\right)^{2}= & U^{T} X_{0} \\
= & \left(\Psi^{T} B_{p}\right)^{2}+\left(\Psi_{1}, \phi_{2}, \ldots, \phi_{p}, \theta_{1}, \theta_{2}, \ldots, \theta_{q}\right)^{T} \\
& \left.\left(y_{p}, y_{p-1}, \ldots, y_{1}, e_{p}, e_{p-1}, \ldots, e_{p+1-q}\right)\right)^{2} \\
& +\left(\left(\phi_{1}, \phi_{2}, \ldots, \phi_{p}, \theta_{1}, \theta_{2}, \ldots, \theta_{q}\right)^{T}\right. \\
& \left.\left(y_{p+2}, y_{p+1}, \ldots, y_{2}, e_{p+2}, e_{p+1}, \ldots, e_{p+2-q}\right)\right)^{2}+\cdots \\
& +\left(\left(\phi_{1}, \phi_{2}, \ldots, \phi_{p}, \theta_{1}, \theta_{2}, \ldots, \theta_{q}\right)^{T}\right. \\
& \left(y_{n+k-2}, y_{n+k-3}, \ldots, y_{n-k-1-p}, e_{n+k-2}, e_{n+k-3}, \ldots\right. \\
& \left.\left.e_{n+k-1-q}\right)\right)^{2} \\
= & \Psi^{T}\left(U U^{T}\right) \Psi
\end{aligned}
$$

such that the likelihood function in equation (8) can be expressed as

$$
L\left(\Psi, \tau \mid S_{n}^{*}\right) \propto \tau^{\frac{(n+k-1)-p}{2}} \exp \left(-\frac{\tau}{2}\left(\sum_{t=p+1}^{n+k-1} y_{t}^{2}-2 \Psi^{T} V+\Psi^{T} W \Psi\right)\right),
$$

where $V=U^{T} X_{0}$ and $W=U U^{T}$.

\subsection{Posterior distribution}

According to suggestion by Broemeling and Shaarawy in [2], the normal-gamma prior of parameters $\Psi$ and $\tau$ is

$$
\begin{aligned}
\xi(\Psi, \tau)=\xi_{1}(\Psi \mid \tau) \cdot \xi_{2}(\tau) \propto \tau^{\frac{p+2 \alpha-2}{2}} & \\
& \exp \left(-\frac{\tau}{2}\left(\Psi^{T} Q \Psi-\Psi^{T} Q \mu-\mu^{T} Q \Psi+\mu^{T} Q \mu+2 \beta\right)\right),
\end{aligned}
$$

where $\xi_{1} \sim N\left(\mu,(\tau Q)^{-1}\right), \xi_{2} \sim G A M(\alpha, \beta), Q$ is a positive definite matrix of the order $(p+q)$, and $\alpha$ and $\beta$ are parameters. 
TheOREM 3.1. The posterior of $\Psi$ and $\tau$ parameters is

$$
\begin{aligned}
\pi\left(\Psi, \tau \mid S_{n}^{*}\right) \propto \tau^{\frac{n+k+2 \alpha-3}{2}} \exp \left(-\frac{\tau}{2}\left(\Psi^{T} P \Psi-\Psi^{T}(V+Q \mu)\right.\right. & \\
& \left.\left.-(V+Q \mu)^{T} \Psi+K\right)\right),
\end{aligned}
$$

where $P=W+Q$ and $K=\sum_{t=p+1}^{n+k-1} y_{t}^{2}+\mu^{T} Q \mu+2 \beta$.

Pr o of. By applying the Bayes theorem, the posterior of $\Psi$ and $\tau$ is

$$
\begin{aligned}
& \pi\left(\Psi, \tau \mid S_{n}^{*}\right) \propto \tau^{\frac{(n+k-1)-p}{2}} \exp \left(-\frac{\tau}{2}\left(\sum_{t=p+1}^{n+k-1} y_{t}^{2}-2 \Psi^{T} V+\Psi^{T} W \Psi\right)\right) \\
& \times \tau^{\frac{p+2 \alpha-2}{2}} \exp \left(-\frac{\tau}{2}\left(\Psi^{T} Q \Psi-\Psi^{T} Q \mu-\mu^{T} Q \Psi+\mu^{T} Q \mu+2 \beta\right)\right) \\
& \propto \quad \tau^{\frac{n+k+2 \alpha-3}{2}} \exp \left(-\frac{\tau}{2}\left(\sum_{t=p+1}^{n+k-1} y_{t}^{2}-2 \Psi^{T} V+\Psi^{T} W \Psi\right.\right. \\
& \left.\left.+\Psi^{T} Q \Psi-\Psi^{T} Q \mu-\mu^{T} Q \Psi+\mu^{T} Q \mu+2 \beta\right)\right) \\
& \propto \quad \tau^{\frac{n+k+2 \alpha-3}{2}} \exp \left(-\frac{\tau}{2}\left(\Psi^{T}(W+Q) \Psi-\Psi^{T} V\right.\right. \\
& \left.\left.-\Psi^{T} Q \mu-\Psi^{T} V-\mu^{T} Q \Psi+\sum_{t=p+1}^{n+k-1} y_{t}^{2}+\mu^{T} Q \mu+2 \beta\right)\right) \\
& \propto \quad \tau^{\frac{n+k+2 \alpha-3}{2}} \exp \left(-\frac{\tau}{2}\left(\Psi^{T}(W+Q) \Psi-\Psi^{T}(V+Q \mu)\right.\right. \\
& \left.\left.-V^{T} \Psi-(Q \mu)^{T} \Psi+\sum_{t=p+1}^{n+k-1} y_{t}^{2}+\mu^{T} Q \mu+2 \beta\right)\right) \\
& \propto \quad \tau^{\frac{n+k+2 \alpha-3}{2}} \exp \left(-\frac{\tau}{2}\left(\Psi^{T}(W+Q) \Psi-\Psi^{T}(V+Q \mu)\right.\right. \\
& \left.\left.-(V+Q \mu)^{T} \Psi+\sum_{t=p+1}^{n+k-1} y_{t}^{2}+\mu^{T} Q \mu+2 \beta\right)\right) \\
& \propto \quad \tau^{\frac{n+k+2 \alpha-3}{2}} \exp \left(-\frac{\tau}{2}\left(\Psi^{T} P \Psi-\Psi^{T}(V+Q \mu)\right.\right. \\
& \left.\left.-(V+Q \mu)^{T} \Psi+K\right)\right) \text {. }
\end{aligned}
$$




\subsection{Marginal posterior distribution}

The marginal posterior distribution is obtained by integrating the posterior distribution in (12).

TheOREM 3.2. The marginal posterior distribution of $\Psi$ is a multivariate $t$-distribution on $(n+k+2 \alpha-1-p)$ degrees of freedom with mode $P^{-1}(V+Q \mu)$ and scale matrix $\left(\frac{n+k+2 \alpha-1-p}{K-(V+Q \mu)^{T} P^{-1}(V+Q \mu)} P\right)^{-1}$ and can be expressed as

$$
\begin{aligned}
\pi\left(\Psi \mid S_{n}^{*}\right) \propto\left((n+k+2 \alpha-1-p)+\left(\Psi-P^{-1}(V+Q \mu)\right)^{T}\right. \\
\left.\quad \frac{n+k+2 \alpha-1-p}{K-(V+Q \mu)^{T} P^{-1}(V+Q \mu)} P\left(\Psi-P^{-1}(V+Q \mu)\right)\right)^{-\frac{n+k+2 \alpha-1}{2}} .
\end{aligned}
$$

Proof. The marginal posterior distribution of $\Psi$ is obtained by integrating the posterior distribution in equation (12) to $\tau$

$$
\begin{aligned}
& \pi\left(\Psi \mid S_{n}^{*}\right)=\int_{0}^{\infty} \pi\left(\Psi, \tau \mid S_{n}^{*}\right) \mathrm{d} \tau=\int_{0}^{\infty} \tau^{\frac{n+k+2 \alpha-3}{2}} \exp \left(-\frac{\tau}{2}\left(\Psi^{T} P \Psi-\Psi^{T}(V+Q \mu)\right.\right. \\
& \left.\left.-(V+Q \mu)^{T} \Psi+K\right)\right) \mathrm{d} \tau \\
& =\int_{0}^{\infty} \tau^{\frac{n+k+2 \alpha-3}{2}} \exp \left(-\frac{\tau}{2}\left(\Psi^{T} P \Psi-\Psi^{T}(V+Q \mu)-(V+Q \mu)^{T} \Psi+K\right)\right) \mathrm{d} \tau \\
& =\int_{0}^{\infty} \tau^{\frac{n+k+2 \alpha-3}{2}} \exp \left(-\frac{\tau}{2}\left(\Psi^{T} P \Psi-\Psi^{T}(V+Q \mu)\right.\right. \\
& \left.\left.-(V+Q \mu)^{T} \Psi+V^{T} P^{-1}(V+Q \mu)-(V+Q \mu)^{T} P^{-1}(V+Q \mu)+K\right)\right) \mathrm{d} \tau \\
& =\int_{0}^{\infty} \tau^{\frac{n+k+2 \alpha-3}{2}} \exp \left(-\frac{\tau}{2}\left(\Psi^{T} P \Psi-\Psi^{T} P P^{-1}(V+Q \mu)\right.\right. \\
& -(V+Q \mu)^{T} P^{-1} P_{1} \Psi+(V+Q \mu)^{T} P^{-1}(V+Q \mu)-(V+Q \mu)^{T} P^{-1} \\
& \times(V+Q \mu)+K)) \mathrm{d} \tau \\
& =\int_{0}^{\infty} \tau^{\frac{n+k+2 \alpha-3}{2}} \exp \left(-\frac{\tau}{2}\left(\Psi^{T} P \Psi-\Psi^{T} P P^{-1}(V+Q \mu)\right.\right. \\
& -(V+Q \mu)^{T}\left(P^{-1}\right)^{T} P \Psi+(V+Q \mu)^{T}\left(P^{-1}\right)^{T}(V+Q \mu)-(V+Q \mu)^{T} \\
& \left.\left.\times P^{-1}(V+Q \mu)+K\right)\right) \mathrm{d} \tau
\end{aligned}
$$


BAYESIAN ESTIMATE OF PARAMETERS FOR ARMA MODEL FORECASTING

$$
\begin{aligned}
= & \int_{0}^{\infty} \tau^{\frac{n+k+2 \alpha-3}{2}} \exp \left(-\frac{\tau}{2}\left(\Psi^{T} P \Psi-\Psi^{T} P P^{-1}(V+Q \mu)\right.\right. \\
& -\left(P^{-1}(V+Q \mu)\right)^{T} P \Psi+\left(P^{-1}(V+Q \mu)\right)(V+Q \mu)-(V+Q \mu)^{T} P^{-1} \\
& \times(V+Q \mu)+K)) \mathrm{d} \tau \\
= & \int_{0}^{\infty} \tau^{\frac{n+k+2 \alpha-3}{2}} \exp \left(-\frac{\tau}{2}\left(\left(\Psi-P^{-1}(V+Q \mu)\right)^{T}\right.\right. \\
& \left.\left.P\left(\Psi-P^{-1}(V+Q \mu)\right)+K-(V+Q \mu)^{T} P^{-1}(V+Q \mu)\right)\right) \mathrm{d} \tau \\
= & \int_{0}^{\infty} \tau^{\frac{n+k+2 \alpha-3}{2}} \exp \left(-\tau\left(\frac{\left(\Psi-P^{-1}(V+Q \mu)\right)^{T}}{2}\right) \mathrm{d} \tau\right. \\
& \left.\frac{P\left(\Psi-P^{-1}(V+Q \mu)\right)+K-(V+Q \mu)^{T} P^{-1}(V+Q \mu)}{2}\right) \mathrm{d} \tau . \\
= & \int_{0}^{\infty} \tau^{\frac{n+k+2 \alpha-1}{2}-1} \exp \left(-\tau\left(\frac{\left(\Psi-P^{-1}(V+Q \mu)\right)^{T}}{P\left(\Psi-P^{-1}(V+Q \mu)\right)+K-(V+Q \mu)^{T} P^{-1}(V+Q \mu)}\right.\right. \\
& \frac{P(V+V)}{2}(V+V)
\end{aligned}
$$

By applying the formula of gamma distribution to the last equation one obtains

$$
\begin{aligned}
\pi\left(\Psi \mid S_{n}^{*}\right) \propto & \left(\frac{\left(\Psi-P^{-1}(V+Q \mu)\right)^{T} P\left(\Psi-P^{-1}(V+Q \mu)\right)+K-}{2}\right. \\
& \frac{-(V+Q \mu)^{T} P^{-1}(V+Q \mu)}{)^{-\frac{n+k+2 \alpha-1}{2}}} \\
\propto & \left(\frac{\left(\Psi-P^{-1}(V+Q \mu)\right)^{T} P\left(\Psi-P^{-1}(V+Q \mu)\right)+K-}{2}\right. \\
& \left.\frac{-(V+Q \mu)^{T} P^{-1}(V+Q \mu)}{-\frac{(n+k+2 \alpha-1-p)+p}{2}}\right)^{\frac{(n+k+2 \alpha-1-p)+p}{2}} \\
& \left.\times\left(\frac{K-(V+Q \mu)^{T} P^{-1}(V+Q \mu)}{2}\right)^{-\frac{(n+k)}{2}}\right)^{\frac{(n+k+2 \alpha-1-p)+p}{2}} \\
& \times\left(\frac{K-(V+Q \mu)^{T} P^{-1}(V+Q \mu)}{2} \times(n+k+2 \alpha-1-p)^{\frac{(n+k+2 \alpha-1-p)+p}{2}}\right. \\
& \times(n+k+2 \alpha-1-p)^{-\frac{(n+k+2 \alpha-1-p)+p}{2}} \times(n+k)
\end{aligned}
$$




$$
\begin{aligned}
& \propto\left(\frac{\left(\Psi-P^{-1}(V+Q \mu)\right)^{T} P\left(\Psi-P^{-1}(V+Q \mu)\right)+K-}{2}\right. \\
& \left.\underline{-(V+Q \mu)^{T} P^{-1}(V+Q \mu)}\right)^{-\frac{(n+k+2 \alpha-1-p)+p}{2}} \\
& \times\left(\frac{K-(V+Q \mu)^{T} P^{-1}(V+Q \mu)}{2}\right)^{\frac{(n+k+2 \alpha-1-p)+p}{2}} \\
& \times(n+k+2 \alpha-1-p)^{-\frac{(n+k+2 \alpha-1-p)+p}{2}} \\
& \propto\left(\frac{\left(\Psi-P^{-1}(V+Q \mu)\right)^{T} P\left(\Psi-P^{-1}(V+Q \mu)\right)+K-}{2}\right. \\
& \left.\underline{-(V+Q \mu)^{T} P^{-1}(V+Q \mu)}\right)^{-\frac{(n+k+2 \alpha-1-p)+p}{2}} \\
& \times\left(\left(\frac{K-(V+Q \mu)^{T} P^{-1}(V+Q \mu)}{2}\right)^{-1}\right)^{-\frac{(n+k+2 \alpha-1-p)+p}{2}} \\
& \times(n+k+2 \alpha-1-p)^{-\frac{(n+k+2 \alpha-1-p)+p}{2}} \\
& \propto\left(\frac{\left(\Psi-P^{-1}(V+Q \mu)\right)^{T} P\left(\Psi-P^{-1}(V+Q \mu)\right)}{K-(V+Q \mu)^{T} P^{-1}(V+Q \mu)}+1\right)^{-\frac{(n+k+2 \alpha-1-p)+p}{2}} \\
& \times(n+k-1-p+2 \alpha)^{-\frac{(n+k-1-p+2 \alpha)+p}{2}} \\
& \propto\left(\frac{\left(\Psi-P^{-1}(V+Q \mu)\right)^{T}(n+k+2 \alpha-1-p) P\left(\Psi-P^{-1}(V+Q \mu)\right)}{K-(V+Q \mu)^{T} P^{-1}(V+Q \mu)}\right. \\
& +(n+k+2 \alpha-1-p))^{-\frac{(n+k-1-p+2 \alpha)+p}{2}} \\
& \propto\left((n+k+2 \alpha-1-p)+l\left(\Psi-P^{-1}(V+Q \mu)\right)^{T}\right. \\
& \left.\times \frac{n+k+2 \alpha-1-p}{K-(V+Q \mu)^{T} P^{-1}(V+Q \mu)} P\left(\Psi-P^{-1}(V+Q \mu)\right)\right)^{-\frac{n+k+2 \alpha-1}{2}} \\
& \propto\left((n+k-1-p+2 \alpha)+\left(\Psi-P^{-1}(V+Q \mu)\right)^{T}\right. \\
& \left.\frac{n+k-1-p+2 \alpha}{K-(V+Q \mu)^{T} P^{-1}(V+Q \mu)} P\left(\Psi-P^{-1}(V+Q \mu)\right)\right)^{-\frac{n+k+2 \alpha-1}{2}} .
\end{aligned}
$$


ThEOREM 3.3. The marginal posterior distribution of $\tau$ is a gamma distribution with parameters $\left(\frac{n+k+2 \alpha-1}{2}, \frac{K-(V+Q \mu)^{T} P^{-1}(V+Q \mu)}{2}\right)$ and is expressed as

$$
\pi\left(\tau \mid S_{n}^{*}\right) \propto \tau^{\frac{n+k+2 \alpha-1}{2}} \exp \left(-\tau\left(\frac{K-(V+Q \mu)^{T} P^{-1}(V+Q \mu)}{2}\right)\right) .
$$

Proof. The marginal posterior distribution of $\tau$ is obtained by integrating the posterior distribution to $\Psi$

$$
\begin{aligned}
& \pi\left(\tau \mid S_{n}^{*}\right)=\int_{-\infty}^{\infty} \pi\left(\Psi, \tau \mid S_{n}^{*}\right) \mathrm{d} \Psi \\
& \propto \int_{-\infty}^{\infty} \tau^{\frac{n+k+2 \alpha-3}{2}} \exp \left(-\frac{\tau}{2}\left(\Psi^{T} P \Psi-2 \Psi^{T}(V+Q \mu)+K\right)\right) \mathrm{d} \Psi \\
& \propto \int_{-\infty}^{\infty} \tau^{\frac{n+k+2 \alpha-3}{2}} \exp \left(-\frac{\tau}{2}\left(\left(\Psi-P^{-1}(V+Q \mu)\right)^{T}\right.\right. \\
& \left.\left.P\left(\Psi-P^{-1}(V+Q \mu)\right)+K-(V+Q \mu)^{T} P^{-1}(V+Q \mu)\right)\right) \mathrm{d} \Psi \\
& \propto \tau^{\frac{n+k+2 \alpha-3}{2}} \exp \left(-\frac{\tau}{2}\left(K-(V+Q \mu)^{T} P^{-1}(V+Q \mu)\right)\right) \\
& \int_{-\infty}^{\infty} \exp \left(\left(\left(\Psi-P^{-1}(V+Q \mu)\right)^{T} P\left(\Psi-P^{-1}(V+Q \mu)\right)\right)\right) d \Psi \\
& \propto \tau^{\frac{n+k+2 \alpha-3}{2}} \exp \left(-\tau\left(\frac{K-(V+Q \mu)^{T} P^{-1}(V+Q \mu)}{2}\right)\right) \\
& \int_{-\infty}^{\infty} \exp \left(\frac { 1 } { 2 } \left(\left(\Psi-P^{-1}(V+Q \mu)\right)^{T}\left(-\tau^{-1} P^{-1}\right)^{-1}\right.\right. \\
& \left.\left.\left(\Psi-P^{-1}(V+Q \mu)\right)\right)\right) \mathrm{d} \Psi \\
& \propto \tau^{\frac{n+k+2 \alpha-1}{2}-1} \exp \left(-\tau\left(\frac{K-(V+Q \mu)^{T} P^{-1}(V+Q \mu)}{2}\right)\right) \times 1 \\
& \propto \tau^{\frac{n+k+2 \alpha-1}{2}-1} \exp \left(-\tau\left(\frac{K-(V+Q \mu)^{T} P^{-1}(V+Q \mu)}{2}\right)\right) .
\end{aligned}
$$


The Bayes estimator of $\Psi$ is mean posterior of the marginal posterior distribution $\pi\left(\Psi \mid S_{n}^{*}\right)$ that is $\hat{\Psi}=P^{-1}(V+Q \mu)$ and the Bayes estimator of $\tau$ is mean posterior of the marginal posterior distribution $\pi\left(\tau \mid S_{n}^{*}\right)$, that is

$$
\hat{\tau}=\left(K-(V+Q \mu)^{T} P^{-1}(V+Q \mu)\right)^{-1}(n+k+2 \alpha-1) .
$$

\section{Conclusions}

This paper mainly studies the Bayesian approach to finding the mathematical expression of the Bayes estimator of $\Psi$ and $\tau$ parameters for ARMA Model Forecasting under normal-gamma prior. Mathematical analysis used to obtain the Bayes estimator is simple such as the repeated integral in calculus and manipulation with matrices in algebra. The Bayes estimator of $\Psi$ is derived using the marginal posterior distribution $\pi\left(\Psi \mid S_{n}^{*}\right)$ which has a multivariate t-distribution, where the Bayes estimator of $\tau$ is derived using the marginal posterior distribution $\pi\left(\tau \mid S_{n}^{*}\right)$ which has a gamma distribution.

\section{REFERENCES}

[1] BAIN, L. J.-ENGELHARDT, M.: Introduction to Probability and Mathematical Statistics (2nd editon). Duxbury Press, Belmont, California, 2006.

[2] BROEMELING, L. D.-SHAARAWY, S.: Time series: a Bayesian analysis in time domain In: Bayesian analysis of Time Series and Dynamic Models (J.C. Spall, ed.), Statistics: Textbooks and Monographs Vol. 94, Marcel Dekker, New York, 1988, pp. 1-21. (Zbl 0714.62082)

[3] FAN, C.-YAO, S.: Bayesian approach for ARMA process and its application, International Business Research 1 (2008), no. 4, 49-55.

[4] KLEIBERGEN, F.- HOEK, H.: Bayesian analysis of ARMA model using non informative prior, Econometric Institute, Erasmus University, Rotterdam, 1996, 1-24.

[5] LIU, S.I.: Comparison of forecasts for ARMA models between a random coefficient approach and a Bayesian approach, Commun. Statist.-Theory Math. 24 (1995), no. 2, 319-333.

[6] POLE, A.-WEST, M.-HARRISON, J.: Applied Bayesian Forecasting and Time Series Analysis. Chapman and Hall, New York, 1994.

[7] RAMACHANDRAN, K. M.-TSOKOS, C. P.: Mathematical Statistics with Applications. Elsevier Academic Press, San Diego, California, 2009.

[8] UTURBEY, W.: Identification of ARMA model by Bayesian methods applied to streamflow data. In: 9th International Conference on Probabilistic Method Applied to Power Systems KTH, 11-15 June 2006, Stockholm, Sweden, 2006, pp. 1-7. 